\title{
A Review of the Performance of Artifact Filtering Algorithms for Cardiopulmonary Resuscitation
}

\author{
Yushun Gong, Bihua Chen and Yongqin Li* \\ School of Biomedical Engineering, \\ Third Military Medical University and Chongqing University, Chongqing, China
}

Submitted October 2012. Accepted for publication March 2013.

\begin{abstract}
Various filtering strategies have been adopted and investigated to suppress the cardiopulmonary resuscitation (CPR) artifact. In this article, two types of artifact removal methods are reviewed: one is the method that removes CPR artifact using only ECG signals, and the other is the method with additional reference signals, such as acceleration, compression depth and transthoracic impedance. After filtering, the signal-to-noise ratio is improved from $0 \mathrm{~dB}$ to greater than $2.8 \mathrm{~dB}$, the sensitivity is increased to $>90 \%$ as recommended by the American Heart Association, whereas the specificity was far from the recommended $95 \%$, which is considered to be the major drawback of the available artifact removal methods. The overall performance of the adaptive filtering methods with additional reference signal outperforms the methods using only ECG signals. Further research should focus on the refinement of artifact filtering methods and the improvement of shock advice algorithms with the presence of CPR.
\end{abstract}

Keywords: cardiac arrest, cardiopulmonary resuscitation, chest compression, ECG, artifact filtering

\section{INTRODUCTION}

Cardiac arrest, represented by a sudden loss of cardiac function, remains to be a major public health problem and a leading cause of death in many parts of the world [1-3]. Most of cardiac arrest events result from unexpected ventricular fibrillation (VF) along with underlying coronary artery diseases and myocardial ischemia [4]. Immediate bystander cardiopulmonary resuscitation (CPR) and early electrical defibrillation are the recommended treatments for VF. Survival rate decreases 3\%-4\% for each minute of defibrillation delay if bystander CPR is provided [5, 6]. If CPR is not

*Corresponding author: Yongqin Li, MSBME, PhD, School of Biomedical Engineering, Third Military Medical University and Chongqing University, 30 Gaotanyan Main Street, Chongqing, China 400038. Phone: (023) 6877-2483. Fax: (023) 6877-1257. E-mail: leeoken@gmail.com. Other authors: johnsongong@163.com; windice1201@sina.com. 
provided, the decrease in survival rate will reach $7 \%-10 \%$ per minute [7]. CPR improves the victim's chance of survival by providing heart and brain circulation. Thus, the latest guidelines from the American Heart Association (AHA) and the European Resuscitation Council (ERC) emphasize the importance of early, uninterrupted chest compression combined with early defibrillation for a successful resuscitation after cardiac arrest $[8,9]$.

However, when CPR is performed, the mechanical activity associated with thoracic compressions and ventilation introduces artifact components into the electrocardiogram (ECG) signals. These artifacts can condition the shock/non-shock decision of an automated external defibrillator (AED) [10]. In order to perform a reliable ECG signal analysis and provide appropriate defibrillation to patients, CPR is mandated to be interrupted during current resuscitation effort. According to Snyder et al. [11], the average time interval required for rhythm analysis was between 5.2 to 28.4 seconds for commercial AEDs, and only one AED could detect a shockable rhythm within 10 seconds. Therefore, both chest compression and ventilation must be interrupted up to 30 seconds to determine whether a disorganized, shockable rhythm has developed for the current commercial AEDs [11]. However, the duration of these "hands-off" intervals adversely affects the rate of restoration of spontaneous circulation (ROSC) [12]. In experimental animals, a 20 seconds conventional "hands-off" interruption of chest compression reduced the likelihood of successful resuscitation by as much as 50\% [13]. Significantly better outcomes were reported if the hands-off intervals were minimized or totally avoided [14]. In a recent multicenter clinical study, Cheskes et al. [15] proved that longer pre-shock and post-shock pauses were independently associated with a decrease in survival to hospital discharge for patients suffering from cardiac arrest and displaying a shockable rhythm. Therefore, the 2010 AHA Guidelines emphasize the minimization of the hands-off interval between the compression and the shock $[16,17]$. If the ECG waveform analysis algorithms (designed to detect a shockable rhythm, predict the likelihood of successful defibrillation, and evaluate the effectiveness of chest compression in current AEDs) can perform well during ongoing chest compression, they may significantly improve the survival rate compared with standard analysis techniques which work during "hands-off" intervals [18-20]. However, reliable and accurate ECG waveform analysis is a difficult task, especially with the presence of CPR artifact, since the frequency of the human ECG signal is largely overlapped with the characteristic frequency of CPR artifact [21]. Meanwhile, the rates and amplitudes of chest compressions and the ventilations vary with time and therefore, the waveform of the ECG signal corrupted by CPR artifact changes with time, which also increases the complexity of rhythm analysis during CPR. Thus, ECG analysis during CPR is a delicate signal processing problem and needs sophisticated adaptive algorithms.

So far, many digital signal processing techniques have been developed to address the problem of cardiac rhythm analysis during uninterrupted CPR, with two major solutions reported in recent studies. One of them features suppressing/eliminating the artifact and reconstruct the fundamental ECG signal using filtering techniques [22-27]. The other 
searches the identifiable components directly in the corrupted ECG signals for cardiac rhythm recognition by advanced algorithms, such as the study by Li et al. [13].

This article reviews the techniques developed for ECG artifact filtering during uninterrupted CPR. The purpose of this study is to compare the advantages of different methods and their efficacies for artifact removal in out-of-hospital cardiac arrest patients.

We organize this paper as follows. Section 2 presents the publication sources and the searched results. Section 3 introduces the characteristics of artifact and the data models used to evaluate the algorithms. Section 4 summarizes the proposed methods designed for artifact filtering during CPR. Section 5 describes the performances and efficacies of different artifact removal methods reported in the literature. In Discussion (section 6), the advantages and disadvantages of different methods are compared. In Limitation (section 7), potential disadvantages of comparison among different datasets and evaluation criterion are addressed. In Conclusion (section 8), a brief outlook of future research direction in this important field is proposed.

\section{METHOD OF LITERATURE SEARCH}

For a systematic review of all the methods developed for CPR artifact removal, we searched different databases, such as NCBI, EBSCOhost, Embase, Medline, SpringerLink, ScienceDirect, Web of Science and IEEEXplore, with the keywords of artifact filter/filtering/removing and cardiopulmonary resuscitation. A total of 122 publications were collected. Table 1 shows the databases and number of publications searched. Two criteria were used to select these references: the artifact removal algorithms developed for CPR artifact filtering, and the experimental or clinical performance of different algorithms applied to human ECG data. Among these publications, 56 engineering and medical papers were used as references according to the criteria. Finally, 8 papers from ScienceDirect, Web of Science and IEEEXplore about Kalman filter [28, 29], independent component analysis (ICA) [30], coherent line removal [31] and adaptive filter [25, 32-34] were described, compared and discussed according to different artifact removal strategies.

Table 1. Databases and number of related publications (selected/total references)

\begin{tabular}{lc}
\hline Database & Number of publications \\
\hline NCBI & $5 / 16$ \\
EBSCOhost & $10 / 22$ \\
Embase & $7 / 8$ \\
Medline & $2 / 3$ \\
Springer Link & $1 / 5$ \\
Science Direct & $11 / 35$ \\
Web of Science & $12 / 17$ \\
IEEEXplore & $8 / 16$
\end{tabular}




\section{ARTIFACT CHARACTERISTICS AND TESTING DATA MODEL 3.1. Artifact Characteristics}

The source of CPR-related artifact during chest compression has not yet been well addressed. According to Fitzgibbon et al. [21], the artifact was predominantly from the electrode-skin interface. The corrupted ECG might also include signals generated by direct impact of the compressions on chest wall and signals generated by the contraction of thoracic muscles. In addition, both the static electricity and equalizing currents between the ECG amplifier and the patient might contribute to the artifact signals that obfuscated ECG rhythm interpretation [35].

Characteristics of CPR-related artifact feature relative high amplitude and large frequency overlap within the frequency bands of the artifact-free ECG. Figure 1 illustrates the ECG tracing and the time frequency representation of the three common cardiac rhythms. The data were existing AED data, recorded from patients who experienced cardiac arrest and CPR, by the investigators in such a manner that subjects cannot be identified directly or through identifiers linked to the subjects. For asystole (Figure 1-A) that represents the state of no contractions of the myocardium and no electrical activity of the heart, a waveless flat line is recorded in the ECG. The signals recorded during compressions can be regarded as the pure artifact produced by CPR. The energy of the artifact is concentrated around the harmonics of the fundamental frequency near $2 \mathrm{~Hz}$ with a bandwidth of approximately $0-20 \mathrm{~Hz}$. For VF signal that represents the uncoordinated contraction of the cardiac muscle of the ventricles, disorganized signal without identifiable QRS complexes is recorded in the ECG, as shown in Figure 1-B. The energy of VF signal lies in the frequency band of $0-18 \mathrm{~Hz}$, which is totally covered by the artifact. For pulseless electrical activity (PEA) (Figure 1-C) that represents a cardiac arrest situation in which a typical QRS complex is at a relative low heart rate, the time-frequency representation shows that a wider bandwidth $(0-40 \mathrm{~Hz})$ QRS complex is overlapped with a continuous-time noise with bandwidth of $0-20 \mathrm{~Hz}$ when the chest is being compressed.

\subsection{Data Model for Testing}

The data used to test the performance of each artifact removal algorithm is usually generated by the superposition of pure CPR artifact and clean ECG signal. As shown in Figure 2, the CPR artifacts collected during asystole are added to human ECG signals recorded from out-of-hospital cardiac arrest patient without compression at a preset signal-to-noise ratio (SNR) level [35]. These artifact-free ECG signals are generally collected during the hands-off interval of CPR, which can be identified by the synchronized recorded reference signals such as acceleration, compression depth or transthoracic impedance [13]. The combined signals with known SNR are filtered by artifact removal algorithms to suppress the CPR artifacts. The processed signals are then evaluated by the restored SNR or validated by a shock/non-shock decision algorithm.

Another reported data model is to feed the uncorrupted human ECG signals into the heart of the animal with preset amplitude, and to record the corrupted ECG signals from the thorax of the animal during CPR [36]. 
(a) Asystole
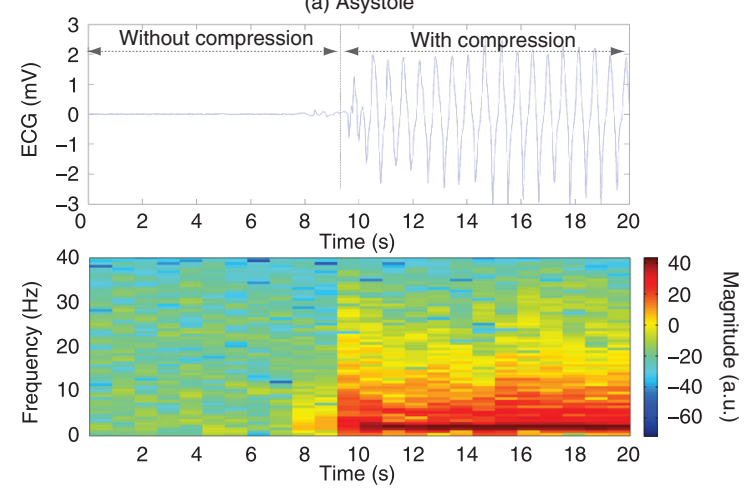

(b) Ventricular fibrillation
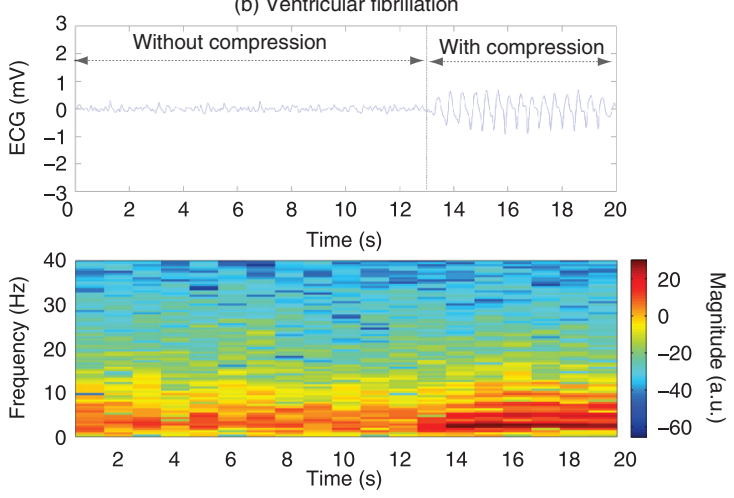

(c) Pulseless electrical
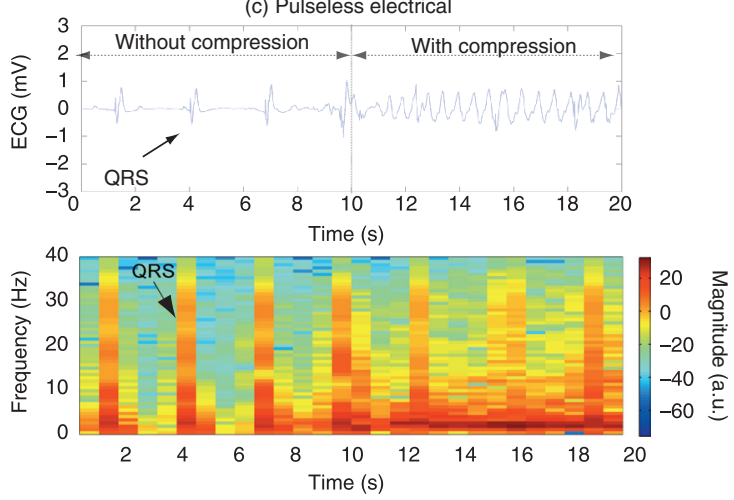

Figure 1. Waveforms of 20 seconds human ECG and the time frequency representation of (a) asystole, (b) ventricular fibrillation, and (c) pulseless electrical activity. 


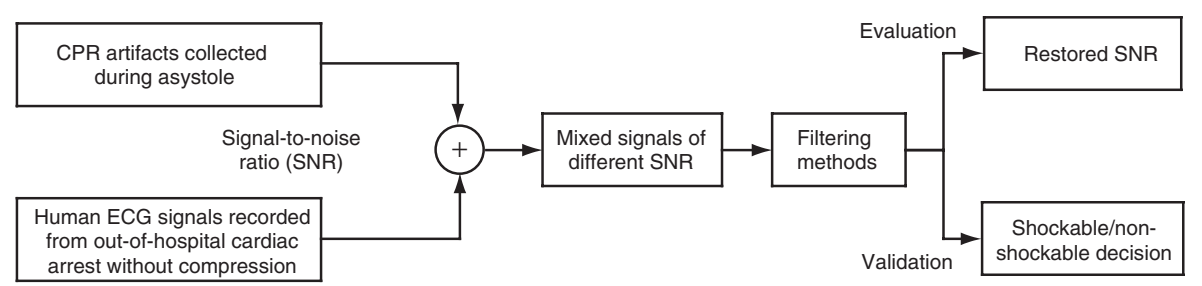

Figure 2. Data model used to evaluate the artifact filtering algorithms.

\section{ALGORITHMS FOR ARTIFACT FILTERING DURING CPR}

In this section, we review the reported algorithms for artifact filtering during CPR. These methods are summarized as the following two categories: 1) methods using only the ECG signals, such as Kalman filter and coherent line removal method using single channel ECG, or ICA that uses multichannel ECGs; 2) methods with additional reference signals besides ECG waveform, such as adaptive filter [33] with single channel reference or MC-RAMP [25] which uses multichannel references.

\subsection{Methods using only ECG Signals}

\subsubsection{Kalman Filter}

The Kalman filter estimates the state of a linear dynamic system in time domain. It is assumed that system state $s_{n}$ in time $n$ is determined by the previous state $s_{n-1}$ and the controlling vector $u_{n}$, and affected by random noise $v_{n}$ :

$$
s_{n}=A_{n} s_{n-1}+B_{n} u_{n}+v_{n}
$$

where $A_{n}$ is the state transition matrix, $B_{n}$ is the matrix specifying the control input, and $v_{n}$ is the process noise of Gaussian distribution with zero mean.

The observation $z_{n}$ of the current system state $s_{n}$ is distorted by noise $w_{n}$ :

$$
z_{n}=M_{n} s_{n}+w_{n}
$$

where $M_{n}$ is the matrix describing the linear operation of taking the observation, and $w_{n}$ is assumed to be Gaussian distribution with zero mean. The current system state can be estimated recursively by the previous state information [37].

In the application of CPR artifact suppression, the CPR artifact presents an almost periodic waveform during chest compressions. Kalman method is appropriate for CPR artifact removal because the recursions provide a numerically fast and adaptive way to estimate CPR artifact from the CPR-corrupted ECG signal.

Rheinberger et al. [29] proposed a Kalman state-space method for removing CPR artifacts in ECG signals. This approach represented the CPR-corrupted signal by a seasonal state-space model, which allowed for a stochastically changing shape of the periodic signal and also coped with time dependent periods. The CPR filtered ECG signals were used to identify the residuals of the Kalman estimation. In this method, the 
transition and observation matrices, as parts of the structural state-space model [38], were updated by reduced maximum likelihood estimation for given observations to reach their optimal values.

Ruiz et al. [28] designed a new CPR suppression method based on Kalman filter, in which the artifact was modeled using the fundamental frequency of the compressions. The frequency of the compression and the relative power content of the artifact were first estimated from the corrupted ECG signal. If the estimated power of the artifact value was lower than a threshold, asystole was excluded and the Kalman filter was used to eliminate the artifact. Then the ECG was fed to the shock/non-shock decision algorithm.

The artifact model is composed of two harmonically related sinusoids:

$$
v_{C P R}(n)=C_{0}(n) \cos \left(\Omega_{0} n+\phi_{0}(n)\right)+K C_{1}(n) \cos \left(2 \Omega_{0} n+\phi_{1}(n)\right)
$$

where $\Omega_{0}$ is the discrete frequency corresponding to the compression frequency, and $K$ is a binary coefficient used to select the second harmonic. Finally, the time-varying amplitudes $C_{0}(n), C_{1}(n)$, and phases of the sinusoidal components $\phi_{0}, \phi_{1}$, are recursively estimated as the state variables of a four-state Kalman filter.

\subsubsection{Independent Component Analysis}

ICA is a statistical and computational technique used to decompose a multichannel mixed signal into independent components [39-42]. It can be represented by a model:

$$
x=D s
$$

where $x=\left\{x^{1}, x^{2}, \ldots, x^{n}\right\}$ is the measured signal from $\mathrm{n}$ channels, $D$ is the mixing matrix, and $s=\left\{s^{1}, s^{2}, \ldots, s^{n}\right\}$ is the signal source which is assumed to be statistically independent. If the mixing matrix $D$ is known, the independent components can be obtained by:

$$
s=D^{-1} x
$$

In the application of CPR artifacts suppression, ECG signals and CPR artifacts are assumed to come from independent sources. Therefore, if the demixing matrix $D^{-1}$ is known, CPR artifacts and artifact-free ECG signals can be separated.

Granegger et al. [30] applied ICA to the CPR-corrupted human ECG signals to reconstruct the ECG signals. In this study, eight channels of human ECG signals were simultaneously recorded in an animal model [36]. In this model, observed signal $x$ collected from eight channels was a mixture of human ECG signals and CPR artifacts. The corrupted signals were assumed to come from independent sources of $s$. These mixed signals were used to compute the inverse matrix in equation (5), which could separate the ECG signals from corrupted signals.

\subsubsection{Coherent Line Removal}

Coherent line removal algorithm removes the periodic signals with sufficiently coupled harmonics [43]. For the reduction of CPR-related artifacts in VF, Amann et al. [31] 
adopted the time-frequency method of coherent line removal algorithm to the specific situations by optimizing its parameters.

In coherent line removal algorithm, the coherent part $a(n)$ of $x(n)=s(n)+a(n)$ is described as

$$
a(n)=\sum_{k=1}^{M} \alpha_{k} m^{k}(n)+\overline{\alpha_{k} m^{k}(n)}
$$

where the over bar denotes complex conjugation and $\alpha_{k}$ are appropriate coefficients, and $m(n)$ is a nearly monochromatic signal,

$$
m(n)=r(n) \exp \left[2 \pi i f_{0}(n)\right]
$$

with slowly varying amplitude $r(n)$ and frequency of compression $f_{0}(n)$, and $i=\sqrt{-1}$. If no CPR artifacts are presented in a time window, $\alpha_{k}$ is set to 0 for all harmonics, where $k=1,2, \ldots, M$.

\subsection{Methods with Additional Reference Signals}

Adaptive filter is a classical algorithm which needs additional references to filter input signals by adjusting the transfer function according to an optimization algorithm driven by an error signal $[44,45]$. Basically, two types of optimization procedures have been employed to adjust the filter coefficients: Least Mean Squares (LMS) and Recursive Least Squares (RLS).

The block diagram of LMS method is shown in Figure 3. In this application, the aim is to estimate the real ECG signal $s(n)$ from the corrupted ECG signal $x(n)$ :

$$
x(n)=s(n)+a(n)
$$

where $a(n)$ is the CPR artifact related noise.

The output of error signal $\hat{s}(n)$ is used to update the adaptive filter coefficient $h(n)$ :

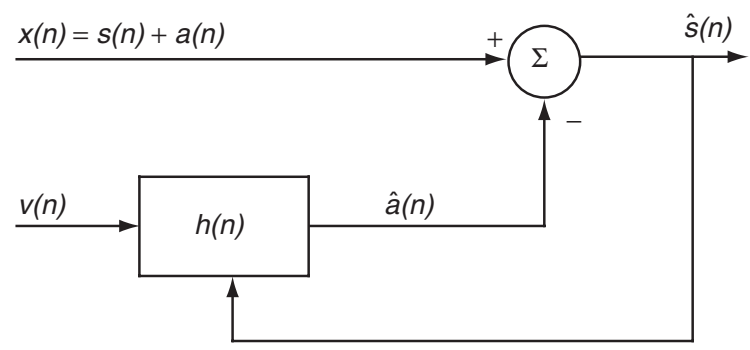

Figure 3. The Least Mean Squares based adaptive filter. 


$$
h(n)=h(n-1)+2 \cdot u \cdot v(n-1) \cdot \hat{s}(n)
$$

where $u$ denotes the step factor. If the reference signal $v(n)$ is related to CPR artifact in the corrupted ECG signal, the output error signal $\hat{s}(n)$ is considered to be the estimation of designed ECG signals.

Irusta et al. [32] estimated the CPR artifact using the frequency of the compressions as the additional reference. In this application, the CPR artifact was assumed to be periodic signal with the frequency of the compression as the fundamental frequency. The time-varying frequency of the compressions was estimated by determining the negative peaks of the compression depth signal. Based on the frequency of the compressions, the CPR artifact was modeled through its Fourier series representation, using harmonics of time-varying amplitude and phases.

During compressions, the artifact is modeled as:

$$
\begin{aligned}
v(n) & =\sum_{k=1}^{N} C_{k}(n) \cos \left[k \phi(n)+\phi_{k}(n)\right] \\
& =\sum_{k=1}^{N} A_{k}(n) \cos [k \phi(n)]+B_{k}(n) \sin [k \phi(n)]
\end{aligned}
$$

where $C_{k}(n)$ and $\phi(n)$ are the amplitude and phase of each harmonic. Based on LMS, $\phi(n)$ is used as the reference input, and the output error $\hat{s}(n)$ is used as the feedback to update coefficients $A_{k}(n)$ and $B_{k}(n)$. The output error $\hat{s}(n)$ is the estimation of designed ECG signals, as shown in Figure 3.

Tan et al. [33] introduced a digital filter which was termed as artifact reduction and tolerant filtering algorithm (ART). In this application, CPR artifact-correlated signals of compression acceleration were collected by the sensor placed beneath the rescuer's hand. These signals were served as the input reference to estimate the real ECG signal by adaptive noise canceller.

Husoy et al. [34] developed a Multichannel Recursive Adaptive Matching Pursuit (MC-RAMP) approach for practical real-time removal of CPR artifact from human ECG signals. The MC-RAMP [46] was an extension of adaptive filter algorithm using multichannel reference signals. In this method, four reference signals including acceleration, compression depth, ECG common and transthoracic impedance were used as reference inputs of adaptive filter. The objective was to find $\hat{a}(n)$ as the best possible estimate of the artifact part of corrupted signal $x(n)$, so that it could be removed by subtracting $\hat{a}(n)$ from $x(n)$. A common solution for adaptive noise canceller is to find the filter coefficients $\underline{h}^{(k)}(n), k=0,1, \ldots, K-1$ ( $K$ is the number of reference signals), at each time $t$ which approaches the minimum of the objective function:

$$
J(n)=\sum_{i=n-L_{2}}^{n+L_{1}}[x(n)-\hat{a}(n)]^{2}
$$


over a rectangular window of size determined through the selection of $L_{1}$ and $L_{2}$, denoting that $L_{1}=0$ and that $L_{1}=L-1$ for convenience.

\section{RESULTS}

The purpose of filtering artifact from CPR-corrupted ECG signals during resuscitation is to enable rhythm analysis during continuous chest compression, thereby increasing the resuscitation success rate by shortening the pre-shock and post-shock pauses of CPR. In order to evaluate the performance of these methods, a large patient dataset is needed and two types of criteria are commonly employed: the restored SNR for corrupted ECG signals constructed by the superposition of artifact-free ECG signals and CPR artifacts, and the improvement of sensitivity and specificity of shock/nonshock decision of an AED for signals recorded from out-of-hospital patients during CPR. The sensitivity was expected to be above $90 \%$ for VF rhythms, and the specificity was expected to be above $95 \%$ for non-shockable rhythms, according to the desired performance goals for arrhythmia analysis algorithms recommended by the AHA task force on AEDs [47]. In this section, we describe the performances of the methods in three segments: (1) performances of algorithms using only the ECG signals, (2) performances of algorithms with additional reference signals, (3) a study to compare different artifact removal methods with the same database and criterion. Table 2 lists the performances of different CPR artifact filtering algorithms presented in the previous section.

\subsection{Algorithms Using only the ECG Signal}

For Kalman filter, Rheinberger et al. [29] used restored SNR to evaluate the performance of the proposed state-space method. Seven porcine asystole ECG signals representing CPR artifacts and seven human artifact-free VF signals were added pairwise with specific SNR. For each of the 49 mixed signals, the restored SNR improved at all SNR settings. Ruiz et al. [28] used the shock/non-shock decision of an AED to evaluate the performance of the proposed four-state Kalman filter. A dataset consisting of 131 shockable and 347 non-shockable episodes was processed by the proposed filtering method. The sensitivity improved from 59\% to $90 \%$ for the detection of a shockable VF, while the specificity decreased from $91 \%$ to $80 \%$ for the detection of a non-shockable rhythm.

For ICA method, Granegger et al. [30] collected 918 corrupted ECG multichannel signals to test the performance of their method. The corrupted signals were obtained by a special animal model. In this model, a catheter was placed in the esophagus, and human ECG signals were fed in close to the dead pig's heart. The corrupted ECG signals were recorded on the thorax during ongoing chest compressions. The sensitivity of the AED's shock/non-shock decision increased from $93.5 \%$ to $99.8 \%$, and the specificity increased from $50.5 \%$ to $83.2 \%$.

For coherent line removal, Amann et al. [31] generated the corrupted ECG signals by mixing 14 human VF signals with 12 CPR artifacts recorded in an animal during asystole with different SNR levels. The performance of the algorithm was tested through 168 episode pairs. For corrupted signals with SNRs of $-20 \mathrm{~dB},-15 \mathrm{~dB},-10 \mathrm{~dB}$, $-5 \mathrm{~dB}$, and $0 \mathrm{~dB}$, the restored SNRs were $9.3 \pm 2.4 \mathrm{~dB}, 9.4 \pm 2.4 \mathrm{~dB}, 9.5 \pm 2.5 \mathrm{~dB}$, $9.3 \pm 2.5 \mathrm{~dB}$, and $8.0 \pm 2.7 \mathrm{~dB}$ after filtering, respectively. 
Table 2. Performances of different CPR artifact filtering algorithms (restored signal to noise ratio (SNR) is given in the form of mean \pm std.)

\begin{tabular}{|c|c|c|c|c|c|}
\hline Data source & Author & Year & Method & Dataset & Results \\
\hline \multirow[t]{4}{*}{$\begin{array}{l}\text { Out-of-hospital } \\
\text { cardiac arrest } \\
\text { patients }\end{array}$} & $\begin{array}{l}\text { Ruiz, } \\
\text { et al. [28] }\end{array}$ & 2008 & $\begin{array}{l}\text { Four-state } \\
\text { Kalman filter }\end{array}$ & $\begin{array}{l}131 \text { shockable and } \\
347 \text { non-shockable } \\
\text { episodes }\end{array}$ & $\begin{array}{l}\text { Sensitivity } \\
91 \% \\
\text { Specificity } \\
80 \%\end{array}$ \\
\hline & $\begin{array}{l}\text { Irusta, } \\
\text { et al. [32] }\end{array}$ & 2009 & $\begin{array}{l}\text { Compression } \\
\text { frequency } \\
\text { based Adaptive } \\
\text { filter }\end{array}$ & $\begin{array}{l}89 \text { shockable and } \\
292 \text { non-shockable }\end{array}$ & $\begin{array}{l}\text { Sensitivity } \\
95.6 \% \\
\text { Specificity } \\
86.4 \%\end{array}$ \\
\hline & $\begin{array}{l}\text { Eilevstjonn, } \\
\text { et al. [25] }\end{array}$ & 2004 & MC-RAMP & $\begin{array}{l}92 \text { shockable } \\
\text { and } 174 \text { non } \\
\text {-shockable } \\
\text { episodes }\end{array}$ & $\begin{array}{l}\text { Sensitivity } \\
96.7 \% \\
\text { Specificity } \\
79.9 \%\end{array}$ \\
\hline & $\begin{array}{l}\text { Tan, } \\
\text { et al. [33] }\end{array}$ & 2008 & ART & $\begin{array}{l}114 \text { shockable } \\
\text { and } 4155 \text { non- } \\
\text { shockable } \\
\text { episodes }\end{array}$ & $\begin{array}{l}\text { Sensitivity } \\
92.1 \% \\
\text { Specificity } \\
90.5 \%\end{array}$ \\
\hline \multirow{4}{*}{$\begin{array}{l}\text { Animal CPR } \\
\text { artifact } \\
\text { mixed with } \\
\text { human ECG }\end{array}$} & $\begin{array}{l}\text { Rheinberger, } \\
\text { et al. [29] }\end{array}$ & 2005 & $\begin{array}{l}\text { Kalman } \\
\text { State-space } \\
\text { method }\end{array}$ & $\begin{array}{l}7 \text { Porcine CPR } \\
\text { artifacts mixed } \\
\text { with } 7 \text { human VF }\end{array}$ & $\begin{array}{l}\text { Restored SNR } \\
\text { from } 0 \mathrm{~dB} \text { to } \\
2.8 \pm 1.4 \mathrm{~dB}\end{array}$ \\
\hline & $\begin{array}{l}\text { Granegger, } \\
\text { et al. }[30]\end{array}$ & 2011 & ICA & $\begin{array}{l}437 \text { shockable } \\
\text { and } 417 \text { non- } \\
\text { shockable human } \\
\text { ECG corrupted by } \\
\text { animal CPR } \\
\text { artifacts }\end{array}$ & $\begin{array}{l}\text { Sensitivity } \\
99.8 \% \\
\text { Specificity } \\
83.2 \%\end{array}$ \\
\hline & $\begin{array}{l}\text { Husoy, } \\
\text { et al. [34] }\end{array}$ & 2002 & MC-RAMP & $\begin{array}{l}24 \text { Animal CPR } \\
\text { artifacts mixed } \\
\text { with } 200 \text { human } \\
\text { VF and } 71 \\
\text { human VT }\end{array}$ & $\begin{array}{l}\text { Restored SNR } \\
\text { from } 0 \mathrm{~dB} \text { to } \\
7.38 \pm 1.78 \mathrm{~dB}\end{array}$ \\
\hline & $\begin{array}{l}\text { Amann, } \\
\text { et al. [31] }\end{array}$ & 2010 & $\begin{array}{l}\text { coherent line } \\
\text { removal }\end{array}$ & $\begin{array}{l}12 \text { Animal CPR } \\
\text { artifacts mixed with } \\
14 \text { human VF }\end{array}$ & $\begin{array}{l}\text { Restored SNR } \\
\text { from } 0 \mathrm{~dB} \text { to } \\
8.0 \pm 2.7 \mathrm{~dB}\end{array}$ \\
\hline
\end{tabular}

\subsection{Algorithms with Additional Reference}

5.2.1. Signals Generated by Mixing Artifact-free Human ECG with Animal CPR Artifact To evaluate the performance of the adaptive filter with additional references, signals generated by combining artifact-free human ECG with animal CPR artifact are used. Husoy at el. [34] used transthoracic impedance, compression depth, ECG common and acceleration as the multiple input reference signals to remove artifact by the proposed 
MC-RAMP. To simulate CPR artifact in corrupted ECG signals, 24 records of animal asystole ECG signals with CPR artifacts were added to 200 records of human VF signals and 71 records of human ventricular tachycardia signals. For signals with SNR of $0 \mathrm{~dB}$, the restored SNRs were ranged from $5.5 \mathrm{~dB}$ to $7.4 \mathrm{~dB}$ with different combinations of these four types of reference signals.

\subsubsection{Signals Recorded from Out-of-hospital Patients}

To validate the clinical performance of adaptive filter with additional reference, Irusta $e t$ al. [32] used the compression frequency as reference to suppress the CPR artifact. The algorithm was tested through 89 shockable and 292 non-shockable ECG samples recorded from out-of-hospital cardiac arrest patients, and the performance was validated using the shock advice algorithm of a commercial AED. The sensitivity and specificity were $97.8 \%$ and $99.0 \%$ with artifact-free ECG signals, respectively, and decreased to $58.4 \%$ and $90.8 \%$ when ECG signals were corrupted by the CPR artifact. After filtering, the sensitivity improved to $95.6 \%$, while the specificity decreased to $86.4 \%$, by using LMS filter.

Tan et al. [33] used compression acceleration as a reference to filter the CPR artifacts. The algorithm was evaluated through 114 shockable and 4,155 non-shockable ECG samples recorded from out-of-hospital patients. Figure 4 and Figure 5 illustrate the waveforms of corrupted, filtered ECG and acceleration signals using the ART filter. For both VF and PEA signals, the CPR artifacts were well suppressed after processing. As a result, a sensitivity of $92.1 \%$ and a specificity of $90.5 \%$ were achieved.

To validate the algorithm of MC-RAMP through the performance of shock/nonshock decision, the reference signals including acceleration, compression depth, ECG common and transthoracic impedance were synchronized and recorded through a modified AED prototype in real resuscitation scenarios by Eilevstjonn et al. [25]. For a patient dataset consisting of 92 shockable and 174 non-shockable episodes, a sensitivity of $96.7 \%$ and a specificity of $79.9 \%$ were achieved, with an increase of approximately $15.0 \%$ and $13.0 \%$, respectively, compared with the unfiltered data.

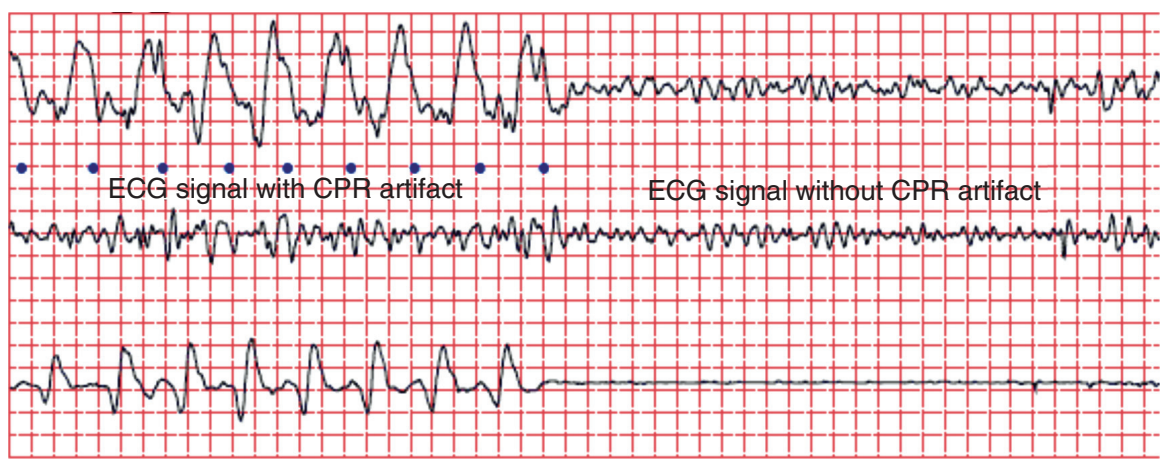

Figure 4. An example of filtering for ventricular fibrillation. The upper trace is the measured ECG signal with/without CPR artifact. The middle trace is the filtered signal. The lower trace is CPR acceleration signal (Reprinted with permission from Tan et al. [33]). 


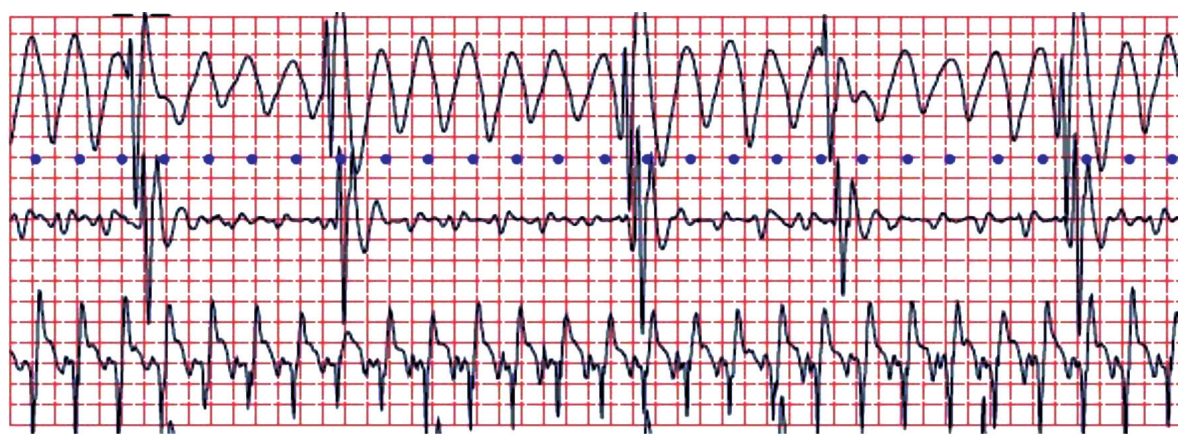

Figure 5. An example of filtering for pulseless electrical activity. The upper trace is the measured ECG signal with CPR artifact. The middle trace is the filtered signal. The lower trace is CPR acceleration signal (Reprinted with permission from Tan et al. [33]).

\subsection{A Study on Comparison of Different Artifact Removal Methods}

To evaluate the efficiency of different artifact removal methods with the same dataset and identical criterion, Werther et al. [48] compared the performance of four different algorithms with signals generated from 395 human artifact-free ECG signals and 13 pure CPR artifacts with corresponding blood pressure serving as reference channels. The human ECG signals were combined with CPR artifacts at different SNR levels ranging from $-10 \mathrm{~dB}$ to $+10 \mathrm{~dB}$. After filtering, CPR artifacts were suppressed and the improvements were significant for all of the four following algorithms: the adaptive matching pursuit algorithm (AF), the regression Kalman state-space model (KF), the motion artifact reduction system using noise cancellation (NC), and the Gabor multiplier (GM). The sensitivity improved from $76.0 \%$ to $100.0 \%, 100.0 \%, 99.0 \%$, and $96.2 \%$ (all above the $90 \%$ requirement for shockable rhythm detection). The specificity, however, was $84.0 \%, 86.8 \%, 83.6 \%$, and $89.7 \%$ for $\mathrm{AF}, \mathrm{KF}, \mathrm{NC}$ and GM, respectively, without significant improvement compared with the $89.3 \%$ specificity for the unfiltered data.

\section{DISCUSSION}

Accurate and prompt detection of shockable rhythms is of vital importance in AEDs. However, reliable detection of ECG rhythms is still a difficult task, especially in the presence of artifacts, such as in the scenario of CPR.

As the Kalman filter estimates CPR artifact from the corrupted ECG signal directly without additional information of reference signals, it can be easily incorporated without additional hardware structure of AED. But Kalman filter requires a priori knowledge of the artifact to evaluate the result recursively [49]. Therefore, the CPR signal is assumed to be a periodic or quasi-periodic signal. However, in practice, CPR artifact may not be a periodic or quasi-periodic signal due to the electrode movement.

Compared with Kalman filter, adaptive filter with a related reference signal needs no a priori statistical knowledge of the CPR artifact [50-52]. It requires one or more 
additional channels to collect reference signals which would increase the complexity of algorithm as well as the structure of AED. When the reference signal comes from another channel that is independent of the ECG signal but correlated with the CPR artifact, the CPR artifact can be suppressed as the reference signal contains useful information about the CPR artifact. Thus, the correlation between reference signal and artifact determines the performance of the filter. However, choosing the appropriate reference signals to suppress CPR artifact is still a challenge that limits the performance of adaptive filter. Even though multichannel references are used in some methods, the improvement is not significant.

The method of ICA can decompose the mixed statistical independent signals, but it needs multichannel signals containing different information of the ECG sources and artifact sources to form up the mixing matrix. When mixed sources are separated, the source of ECG must be identified. However, correctly identifying ECG sources is still a challenge. Besides, ICA is not capable of dealing with asystolic signals, since asystolic ECG signals do not represent an identifiable source if electrical heart activity ceases. When asystole occurs, ICA would fail to correctly reconstruct the signals and could also misclassify the extracted component.

The performance of these methods showed that the sensitivity increased to more than $90 \%$, above the $90 \%$ level recommended by AHA Task Force on AED, while the specificity ranged from $79.9 \%$ to $90.5 \%$, far below the recommended $95 \%$. Compared with the published results of sensitivity, ICA method outperformed others, with a value of $99.8 \%$. In terms of specificity, the adaptive filter proposed by Tan et al. performed better than other methods, with a value of $90.5 \%$.

These results show that although the sensitivity of the shock advice algorithm is greatly improved, the specificity is still below the recommended level especially for PEA and asystole, perhaps due to the electrical activity in the heart and thorax muscle or the movement of the electrode pads during compression [53, 54]. Therefore, the filtered signals may still contain residual components of artifact, as shown in figure 5, which lies in the same frequency band as VF signal (Figure 1-B and Figure 1-C). These residual artifact components lead to an inappropriate analysis and reduce the specificity of AED. Besides, lack of artifact-related components in the reference signal for the adaptive filter and/or limitation of shock advice algorithms may also result in insufficient specificity. Although some algorithms have been successfully incoporated in commercial AEDs and can display filtered ECG signals during CPR, the result can only serve as a reference to help physicians in making a decision, and the filtering technique has not been employed for automate rhythm analysis. Insufficient specificity may cause inappropriate shock delivery to patients with non-shockable rhythms, which may be the major reason why the clinical use of filtering devices for rhythm analysis during CPR has not been supported and accepted [55].

\section{LIMITATIONS}

Different datasets were used to test the algorithms summarized in this review. Some are generated by the linear superposition of animal CPR artifact and human artifact-free ECG signal, whereas the others were recorded from the out-of-hospital patients. The use of different datasets would reduce the reliability of comparison among different 
methods. In addition, using different criteria for evaluation in different papers is another limitation. The criterion of restored SNR is an engineering standard which is calculated as the ratio of the signal and noise powers. The sensitivity and specificity are clinical standards mainly concerning the output of an AED. Whether a great improvement in SNR would achieve high values of sensitivity and specificity has not been established. Moreover, the shock advice algorithms used in these methods are different, which also reduce the reliability of direct comparison among different methods based on sensitivity and specificity.

\section{CONCLUSION}

Removal of CPR-related artifact from corrupted ECG signals could enable continuous detection of rhythm changes and estimation of the probability of defibrillation success. This would avoid the "hands-off" analysis intervals which diminish the cardiac perfusion and thus lower the chance for a successful defibrillation attempt. The artifact filtering techniques proposed for ECG rhythm analysis during CPR are promising but still facing challenges, especially for the non-shockable rhythms. Filtering methods with reference signals outperform those methods without references if the reference signals are well correlated with artifacts. Further studies are therefore recommended to improve the performance of adaptive filters by choosing appropriate reference signals, and analyzing the interaction between the filtering method and shock advice algorithm of AED to improve the accuracy of non-shock decision. Investigations should also focus on both the refinement of artifact filtering algorithms and the improvement of shock advice algorithms with the presence of CPR [56].

\section{CONFLICT OF INTEREST}

The authors indicated no potential conflicts of interest.

\section{ACKNOWLEDGMENTS}

This project was supported by the National Nature Science Foundation of China (NSFC31070884, NSFC81271656), the Foundation for the Author of National Excellent Doctoral Dissertation of China (FANEDD 201060), and the Natural Science Foundation Project of Chongqing (CQCSTC2010BB5023).

\section{REFERENCES}

[1] McNally B, Robb R, Mehta M, Vellano K, Valderrama A L, Yoon P W, Sasson C, Crouch A, Perez A B, Merritt R, Kellermann A. Out-of-hospital cardiac arrest surveillance - cardiac arrest registry to enhance survival (CARES), United States, October 1, 2005-December 31, 2010. Morbidity and Mortality Weekly Report, 2011, 60(8):1-19.

[2] Crampton R. The problem of cardiac arrest in the community. The American journal of emergency medicine, 1984, 2(3):204-9.

[3] Mehra R. Global public health problem of sudden cardiac death. Journal of Electrocardiology, 2007, 40(6):s118-s122.

[4] Luu M, Stevenson WG, Stevenson LW, Baron K, Walden J. Diverse mechanisms of unexpected cardiac arrest in advanced heart failure. Circulation, 1989, 80(6):1675-80.

[5] Valenzuela TD, Roe DJ, Cretin S, Spaite DW, Larsen MP. Estimating effectiveness of cardiac arrest interventions: a logistic regression survival model. Circulation, 1997, 96(10):3308-13. 
[6] Chan PS, Krumholz HM, Nichol G, Nallamothu BK. Delayed time to defibrillation after in-hospital cardiac arrest. New England Journal of Medicine, 2008, 358(1):9-17.

[7] Larsen MP, Eisenberg MS, Cummins RO, Hallstrom AP. Predicting survival from out-of-hospital cardiac arrest: a graphic model. Annals of emergency medicine, 1993, 22(11):1652-8.

[8] Link MS, Atkins DL, Passman RS, Halperin HR, Samson RA, white RD, Cudnik MT, Berg MD, Kudenchuk PJ, Kerber RE. Part6: Electrical therapies automated external defibrillators, defibrillation, cardioversion, and pacing 2010 American Heart Association Guidelines for Cardiopulmonary Resuscitation and Emergency Cardiovascular Care. Circulation, 2010, 112(18):s706-s719.

[9] Deakin CD, Nolan JP, Sunde K, Koster RW. European Resuscitation Council guidelines for resuscitation 2010 section 3. Electrical therapies: automated external defibrillators, defibrillation, cardioversion and pacing. Resuscitation, 2010, 81(10):1293-304.

[10] Yu T, Weil MH, Tang W, Sun S, Klouche K, Povoas H, Bisera J. Adverse outcomes of interrupted precordial compression during automated defibrillation. Circulation, 2002, 106:368-72.

[11] Snyder D, Morgan C. Wide variation in cardiopulmonary resuscitation interruption internals among commercially available automated external defibrillators may affect survival despite high defibrillation efficacy. Critical Care Medicine, 2004, 32(9):s421-4.

[12] Valenzuela TD, Kern KB, Clark LL, Berg RA, Berg MD, Berg DD, Hilwig RW, Otto CW, Newburn D, Ewy GA. Interruptions of chest compressions during emergency medical systems resuscitation. Circulation, 2005, 112(9):1259-65.

[13] Li Y, Bisera J, Geheb F, Tang W, Weil MH. Identifying potentially shockable rhythms without interrupting cardiopulmonary resuscitation. Critical Care Medicine, 2008, 36(1):198-203.

[14] Eftestol T, Sunde K, Steen PA. Effects of interrupting precordial compressions on the calculated probability of defibrillation success during out-of-hospital cardiac arrest. Circulation, 2002, 105(9):2270-3.

[15] Cheskes S, Schmicker RH, Christenson J, Salcido DD, Rea T, Powell J, Edelson DP, Sell R, May S, Menegazzi JJ, Van Ottingham L, Olsufka M, Pennington S, Simonini J, Berg RA, Stiell I, Idris A, Bigham B, Morrison L. Perishock Pause An Independent Predictor of Survival From Out-of-Hospital Shockable Cardiac Arrest. Circulation, 2011, 124(1): 58-66.

[16] Yu T, Ristagno G, Li Y, Bisera J, Weil MH, Tang W. The resuscitation blanket: A useful tool for "hands-on" defibrillation. Resuscitation, 2010, 81(2):230-5.

[17] Ristagno G, Tang W, Russell JK, Jorgenson D, Wang H, Sun S, Weil MH. Minimal interruption of cardiopulmonary resuscitation for single shock as mandated by automated external defibrillations does not compromise outcomes in a porcine model of cardiac arrest and resuscitation. Critical Care Medicine, 2008, 36(11):3048-53.

[18] Amann A, Achleitner U, Antretter H, Bonatti JO, Krismer AC, Lindner KH, Rieder J, Wenzel V, Voelckel WG, Strohmenger HU. Analysing ventricular fibrillation ECG-signals and predicting defibrillation success during cardiopulmonary resuscitation employing N(alpha)-histograms. Resuscitation, 2001, 50(1):77-85.

[19] Hamprecht FA, Achleitner U, Krismer AC, Lindner KH, Wenzel V, Strohmenger HU, Thiel W, van Gunsteren WF, Amann A. Fibrillation power, an alternative method of ECG spectral analysis for prediction of countershock success in a porcine model of ventricular fibrillation. Resuscitation, 2001, 50(3):287-96.

[20] Amann A, Mayr G, Strohmenger HU. N( $\alpha$ )-histogram analysis of the ventricular fibrillation ECGsignal as predictor of countershock success. Chaos, Solitons \& Fractals, 2000, 11(8):1205-12.

[21] Fitzgibbon E, Berger R, Tsitlik J, Halperin HR. Determination of the noise source in the electrocardiogram during cardiopulmonary resuscitation. Critical Care Medicine, 2002, 30(4):148-53.

[22] Werther T, Klotz A, Kracher G, Baubin M, Feichtinger HG, Gilly H, Amann A. CPR artifact removal in ventricular fibrillation ECG signals using Gabor multipliers. IEEE Transactions On Biomedical Engineering, 2009, 56(6):320-7. 
[23] Aase SO, Eftestol T, Husoy JH, Sunde K, Steen PA. CPR artifact removal from human ECG using optimal multichannel filtering. IEEE Transactions on Biomedical Engineering, 2000, 47(11):1440-9.

[24] Rheinberger K, Steinberger T, Unterkofler K, Baubin M, Klotz A, Amann A. Removal of CPR artifacts from the ventricular fibrillation ECG by adaptive regression on lagged reference signals. IEEE Transactions on Biomedical Engineering, 2008, 55(1):130-7.

[25] Eilevstjonn J, Eftestol T, Aase SO, Myklebust H, Husoy JH, Steen PA. Feasibility of shock advice analysis during CPR through removal of CPR artefacts from the human ECG. Resuscitation, 2004, 61(2):131-41.

[26] Aramendi E, Ayala U, Irusta U, Alonso E, Eftestol T, Kramer-Johansen J. Suppression of the cardiopulmonary resuscitation artefacts using the instantaneous chest compression rate extracted from the thoracic impedance. Resuscitation, 2012, 83(6):692-8.

[27] Tong S, Bezerianos A, Paul J, Zhu Y, Thakor N. Removal of ECG interference from the EEG recordings in small animals using independent component analysis. Journal of Neuroscience Methods, 2001, 108(1):11-7.

[28] Ruiz de Gauna S, Ruiz J, Irusta U, Aramendi E, Eftestol T, Kramer-Johansen J. A method to remove CPR artefacts from human ECG using only the recorded ECG. Resuscitation, 2008, 76(2):271-8.

[29] Rheinberger K, Baubin M, Unterkofler K, Amann A. Removal of Resuscitation Artefacts from Ventricular Fibrillation ECG Signals Using Kalman Methods. Computers in Cardiology, 2005, 32:555-8.

[30] Granegger M, Werther T, Gilly H. Use of independent component analysis for reducing CPR artefacts in human emergency ECGs. Resuscitation, 2011, 82(1):79-84.

[31] Amann A, Klotz A, Niederklapfer T, Kupferthaler A, Werther T, Granegger M, Lederer W, Baubin M, Lingnau W. Reduction of CPR artifacts in the ventricular fibrillation ECG by coherent line removal. Biomedical Engineering Online, 2010, 9:2.

[32] Irusta U, Ruiz J, de Gauna SR, Eftestol T, Kramer-Johansen J. A Least Mean-Square Filter for the estimation of the cardiopulmonary resuscitation artifact based on the frequency of the compressions. IEEE Transactions on Biomedical Engineering, 2009, 56(4): 1052-62.

[33] Tan Q, Freeman GA, Geheb F, Bisera J. Electrocardiographic analysis during uninterrupted cardiopulmonary resuscitation. Critical Care Medicine, 2008, 36(11):s409-12.

[34] Husoy JH, Eilevstjonn J, Eftestol T, Aase SO, Myklebust H, Steen PA. Removal of cardiopulmonary resuscitation artefacts from human ECG using an efficient matching pursuit-like algorithm. IEEE Transactions on Biomedical Engineering, 2002, 49(11):1287-98.

[35] Langhelle A, Eftestol T, Myklebust H, Eriksen M, Holten BT, Steen PA. Reducing CPR artefacts in ventricular fibrillation in vitro. Resuscitation, 2001, 48(3) :279-91.

[36] Granegger M, Werther T, Roehrich M, Losert U, Gilly H. Human ECGs corrupted with real CPR artefacts in an animal model: generating a database to evaluate and refine algorithms for eliminating CPR artifacts. Resuscitation, 2010, 81(6):730-6.

[37] Kalman RE. A new approach to linear filtering and prediction problems. Transaction of the ASME: Journal of Basic Engineering, 1960, 82:34-45.

[38] Harvey AC. Forecasting, Structural Time Series Models and the Kalman Filter. Cambridge University Press, 1989.

[39] Chawla MPS. PCA and ICA processing methods for removal of artifacts and noise in electrocardiograms: A survey and comparison. Applied Soft Computing, 2011, 11(2):2216-26.

[40] Cheng W, Lee S, Zhang Z, He Z. Independent component analysis based source number estimation and its comparison for mechanical systems. Journal of Sound and Vibration, 2012, 331(23):5153-67.

[41] Deng W, Liu Y, Hu J, Guo J. The small sample size problem of ICA: A comparative study and analysis. Pattern Recognition, 2012, 45(12):4438-50.

[42] De Vos M, Nion D, Van Huffel S, De Lathauwer L. A combination of parallel factor and independent component analysis. Signal Processing, 2012, 92(12):2990-99. 
[43] Sintes AM, Schutz BF. Coherent line removal: filtering out harmonically related line interference from experimental data, with applications to gravitational wave detectors. Phys Rev. D, 1998, 58:122003.

[44] Abadi MSE, Far AM. A unified framework for adaptive filter algorithms with variable step-size. Computer and Electrical Engineering, 2008, 34(3):232-49.

[45] Ho KC, Ching PC. Split filter structures for LMS adaptive filtering. Signal Processing, 1995, 46:255-66.

[46] Husoy JH and Ommundsen J. A novel algorithm for adaptive filters based on optimum selective update of coefficients, in Proc. Applied Electronics, Plzen, Czech Republic, 2001:114-121.

[47] Kerber RE, Becker LB, Bourland JD, Cummins RO, Hallstrom AP, Michos MB, Nichol G, Ornato JP, Thies WH, White RD, Zuckerman BD. Automatic external defibrillators for public access defibrillation: recommendations for specifying and reporting arrhythmia analysis algorithm performance, incorporating new waveforms and enhancing safety. A statement for health professionals from the American Heart Association Task Force on automatic external defibrillation subcommittee on AED safety and efficacy. Circulation, 1997, 95(6):1677-82.

[48] Werther T, Klotz A, Granegger M, Baubin M, Feichtinger HG, Amann A, Gilly H. Strong corruption of electrocardiograms caused by cardiopulmonary resuscitation reduces efficiency of two-channel methods for removing motion artifacts in non-shockable rhythms. Resuscitation, 2009, 80(11):1301-7.

[49] Auger F, Gueerero JM, Hilairet M, Katsura S, Monmasson E, Orlowska-Kowalska T. Introduction to the special section on industrial applications and implementation issues of the Kalman filter. IEEE Transactions on Industrial Electronics, 2012, 59(11):4165-68.

[50] Silva L, Lee J, Mark RG. Signal quality estimation with multichannel adaptive filtering in intensive care settings. IEEE Transactions on Biomedical Engineering, 2012, 59(9):2476-85.

[51] Lee J, Song MH, Shin DG, Lee KJ. Event synchronous adaptive filter based atrial activity estimation in single-lead atrial fibrillation electrocardiograms. Medical \& Biological Engineering \& Computing, 2012, 50(8):801-11.

[52] Sanei S, Lee TK, Abolghasemi V. A new adaptive line enhancer Based on singular spectrum analysis. IEEE Transactions on Biomedical Engineering, 2012, 69(2):428-34.

[53] Li Y, Tang W. Techniques for artefact filtering from chest compression corrupted ECG signals: Good, but not enough. Resuscitation, 2009, 80(11):1219-20.

[54] Lin LY, Lo MT, Chiang WC. A new way to analyze resuscitation quality by reviewing automatic external defibrillator data. Resuscitation, 2012, 83(2):171-6.

[55] Sayre MR, Koster RW, Botha M, Cave DM, Cudnik MT, Handley AJ, Hatanaka T, Hazinski MF, Jacobs I, Monsieurs K, Morley PT, Nolan JP, Travers AH. Part5: Adult basic life support 2010 international consensus on cardiopulmonary resuscitation and emergency cardiovascular care science with treatment recommendations. Circulation, 2010, 122(16):8298-324.

[56] Li Y, Bisera J, Weil MH, Tang W. An algorithm used for ventricular fibrillation detection without interrupting chest compression. IEEE Transactions on Biomedical Engineering, 2012, 59(1):78-86. 


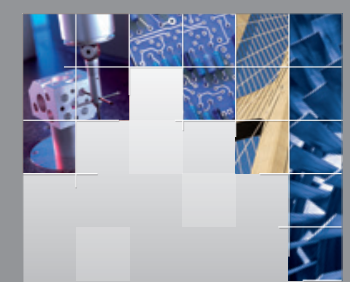

\section{Enfincering}
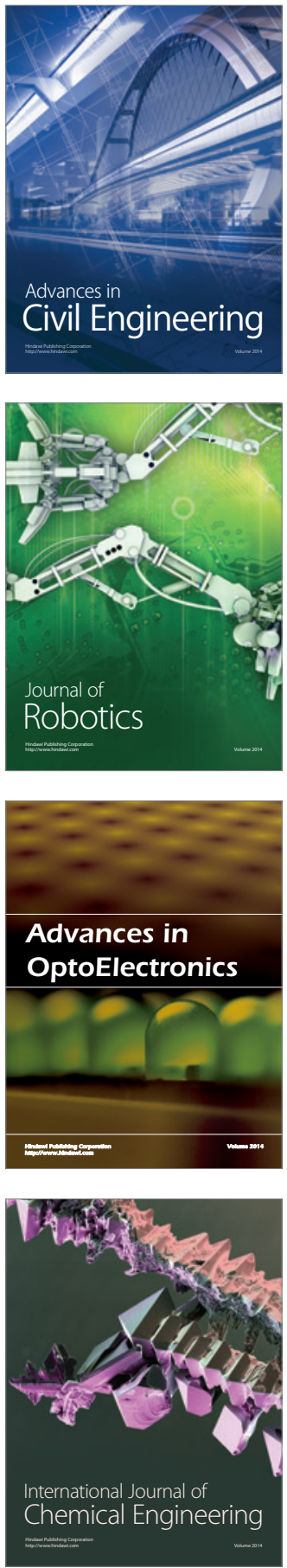

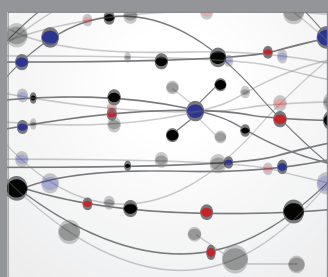

The Scientific World Journal

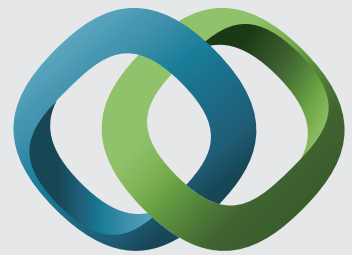

\section{Hindawi}

Submit your manuscripts at

http://www.hindawi.com
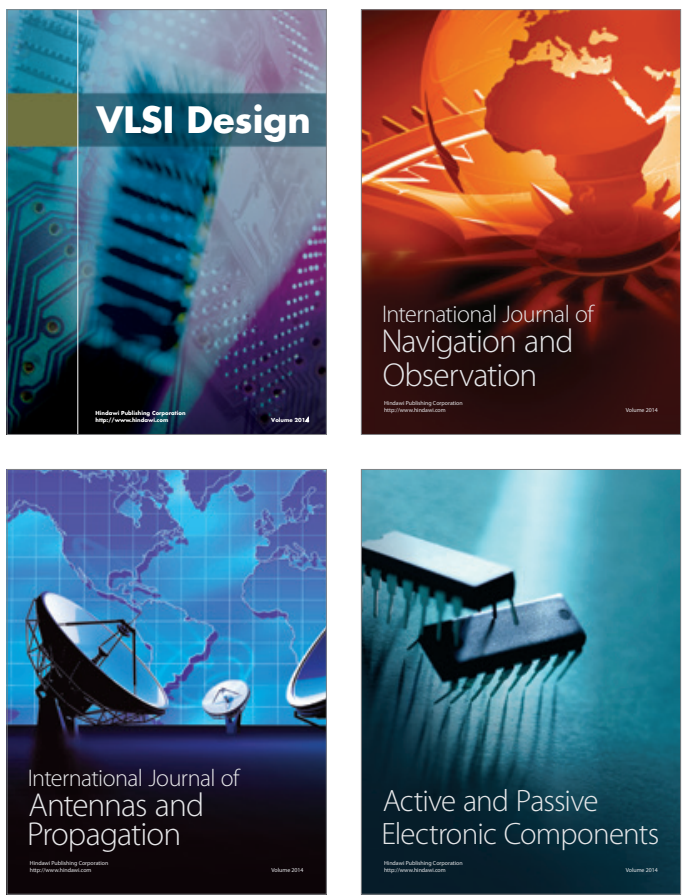
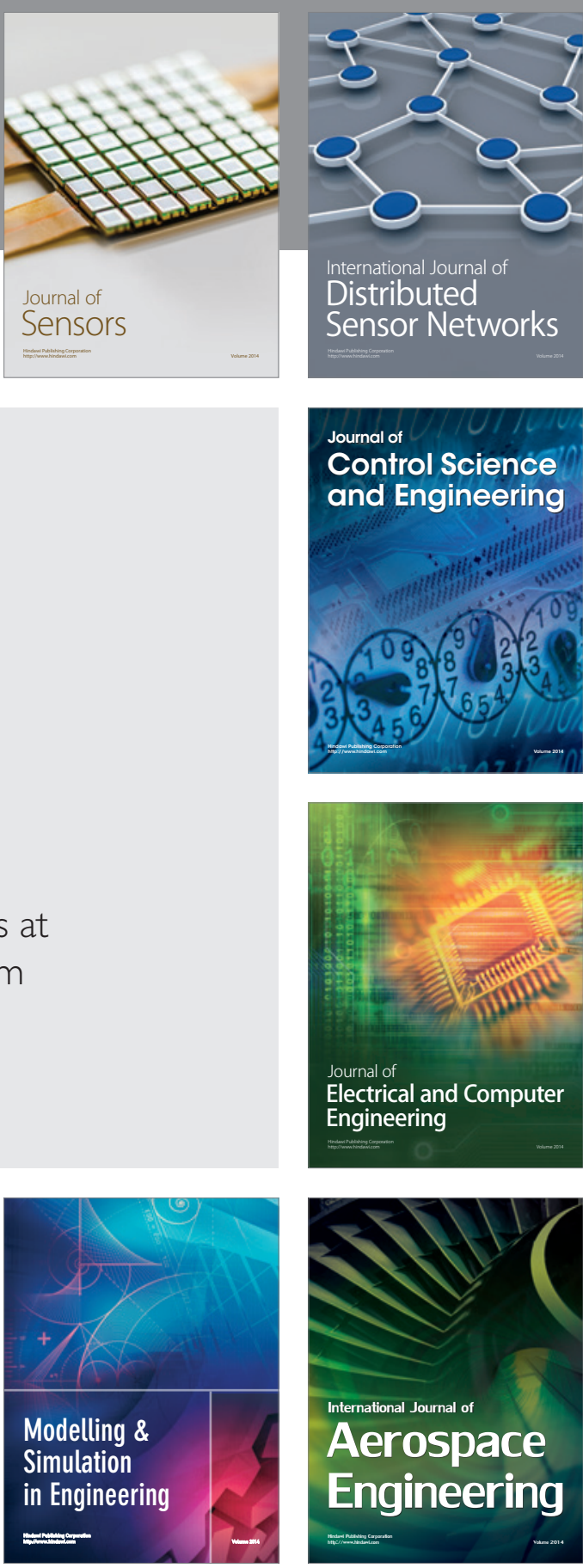

International Journal of

Distributed

Sensor Networks

Journal of

Control Science

and Engineering
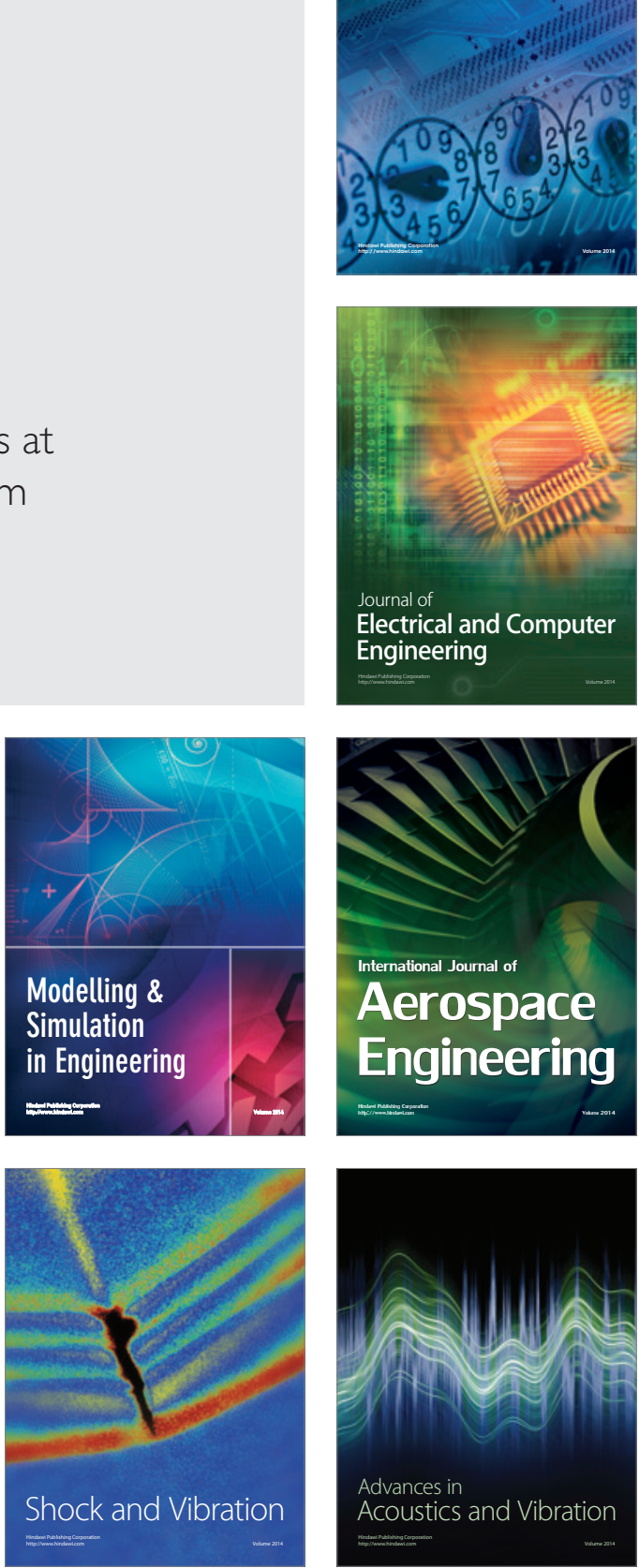